Ann. Abeille, Ig66, 9 (I), 37-45.

\title{
ÉTUDE DE LA CONTAMINATION EXTERNE DE L'ABEILLE APIS MELLIFICA ET DE SON MILIEU PAR UN RADIO-ISOTOPE INTRODUIT DANS LA NOURRITURE
}

\author{
Ph. DOUAULT \\ Station de Recherches sur l'Abeille et les Insectes Sociaux, \\ 91 - Bures-sur-Yvette.
}

\section{SOMMAIRE}

L'auteur étudie la pollution externe de l'abeille, celle-ci étant nourrie soit avec un aliment solide (candi à reine) soit avec un aliment liquide (sirop de sucre). Cette nourriture est marquée au moyen d'un radio-isotope, le phosphore-32, sous la forme de phosphate de sodium.

Les résultats obtenus montrent que, contrairement à ce que l'on pensait, la contamination externe de l'abeille est faible dans les deux cas. La contamination des cagettes dans lesquelles vivent les abeilles pendant toute la durée de l'expérience est également faible. Un examen des différents organes, et en particulier des pattes, montre bien que la radioactivité est localisée à l'intérieur du corps de l'insecte.

\section{INTRODUCTION}

Les échanges de nourriture entre les abeilles d'une même ruche ont déjà été étudiés au moyen des radio-isotopes mais il ne semble pas qu'on se soit suffisamment penché sur le problème de la contamination externe, facteur qui pourtant risquerait de troubler quelque peu les résultats.

Nixon et RibBands (1952) ont fait ingérer aux ouvrières une nourriture liquide sucrée marquée par le phosphore-32; ils ont constaté que sur les quelques 20000 abeilles d'une ruche, $60 \mathrm{p}$. Ioo devenaient radioactives au bout de $24 \mathrm{~h}$ par suite d'échanges.

OERTEL et al. (I953) ont observé de même les échanges entre les mâles et les ouvrières d'abeilles à l'aide d'une nourriture marquée. CouRTOIS et LECOMTE (I958) ont constaté qu'au bout de $36 \mathrm{~h}$, roo p. Ioo des abeilles d'une même ruche étaient radioactives après ingestion d'or-198. 
DEIVERT-SALLERON (I962) a étudié les échanges de notirriture: Io entre ouvrières sœurs et entre ouvrières étrangères, c'est-à-dire provenant de deux ruches différentes ; $2^{\circ}$ entre les mâles et les ouvrières; $3^{\circ}$ entre les reines et les ouvrières.

La radioactivité transmise d'un individu à l'autre a été dans tous les cas attribuée à une contamination interne. Les méthodes de marquage variant peu, les abeilles recevant les premières la nourriture marquée, la communiquent par la langue à leurs congénères; cette nourriture vient donc directement du jabot. L'éventualité d'une contamination externe n'a pas été souvent mentionnée.

Nous avons voulu estimer l'importance des contaminations en utilisant deux sortes de nourriture : ${ }^{0}$ solide : candi à reine $; 2^{\circ}$ liquide : sirop de sucre.

\section{MA'TÉRIEL E'T MÉTHODES}

I. Abeilles.

Les abeilles ouvrières que nous avons utilisées dans ces expériences étaient prélevées au hasard sur un cadre provenant d'une même ruche. Nous évitions, dans tous lés cas, d'utiliser un cadre de couvain afin de ne pas risfuer la capture d'abeilles naissantes ou trop jeunes.

\section{Cagettes.}

Les cagettes étaient conservées dans une étuve réglée à $30^{\circ} \mathrm{C}$.

Les cagettes étaient du type Iiebefeld, matériel déjầ utilisé depuis longtemps au laboratoire. Elles se présentent sous la forme d'un cadre de bois compris ent re deux parois vitrées de $13 \times 16 \mathrm{~cm}$; une languette de cire gaufrée est suspendue à l'intérieur de la cagette.

Chaque cagette contenait I $_{5}$ à 20 abeilles suivant le cas.

\section{Nourriture.}

La nourriture était présentée sous deux formes :

$I^{\circ}$ Candi à reine solide, habituellement utilisé pour les élevages, se composant d'un mélange de sucre glace et de miel, tel que le produit fini soit de consistance analogue au mastic.

$2^{\circ}$ Sirop : mélange de miel et d'eau à poids égaux.

\section{Nourrisseur.}

Le nourrisseur utilisé dans le cas de la nourriture solidc était une boîte carrée en matière plastique de $4 \mathrm{~cm} \times 2,7 \mathrm{~cm}$. Dans le cas de la nourriture liquide, le nourrisseur se présentait sous la forme d'une cupule de verre de 0,5 de dianètre et $0,7 \mathrm{~cm}$ de profondeur, disposée sur un socle. Lai cupule était entourée d'un grillage métallique à fines mailles afin que l'abeille puisse s'agripper par les pattes et se maintenir au bord de la cupule. Le liquide était suffisamment bas dans le récipient pour que l'abeille puisse l'atteindre facilement avec sa langue, sans se souiller cependant les pattes.

\section{Isotope utilisé.}

L'isotope que nous avons employé était le phosphore-32 fourni par le Commissariat à l'Énergie atomique sous la forme de phosphate monosodique.

L'isotope était incorporé à la nourriture : $1^{0}$ solide, par introduction avant le refroidissement du candi et pénétration par un soigneux malaxage de la pâte ; $2^{\circ}$ liquide, par adjonction de la solution marquée au sirop de sucre. Par mesure de sécurité, les manipulations étaient faites dans une boîte à gants. Nous avons introduit dans la nourriture une quantité suffisante de matière radioactive pour que charque abeille puisse absorber à peu près un microcurie.

\section{Méthode de comptage.}

L'appareil de comptage utilisé était un compteur Geiger-Müller du type cloche sous château de plomb. Nous avons travaillé à une tension constante de I 475 volts. Le bruit de fond de l'appareil variait de 12 à 16 coups par minute. 
Au début de chaque série de comptage, le mouvement propre de l'appareil était déterminé par une surveillance du bruit de fond d'au moins ro mn. Tous les ro comptages, une mesure de bruit de fond était effectuée, pour voir s'il n'y avait pas eu contamination de l'ensemble de comptage.

Les différentes pièces que nous avons étudiées étaient disposées sous la cellule en s'efforçant de respecter des conditions de géométrie comparables.

Quand elles se trouvaient être de taille trop importante, nous avons, chaque fois que la chose était possible, procédé à un lessivage des parties éventuellement contaminées avec un tampon de coton imbibé d'eau ; ce tampon a fait l'objet du comptage.

Enfin quand le matériel était de taille trop grande pour être introduit sous le compteur G.-M. et ne pouvait être lessivé, nous avons utilisé un intégrateur IPAB 2 B à sondes bêta-gamma constitué par 2 compteurs Geiger-Müller du type hallogène, 3 B 17. Cet appareil et couramment utilisé pour la détection des radio-isotopes de moyenne énergie, le bruit de fond de cet appareil étant de 2 chocs par seconde.

\section{Notation des résultats.}

Les résultats consignés dans ce travail sont exprimés en coups nets, c'est-à-dire, le nombre de coups par minute lus sur le compteur, après déduction du bruit de fond.

Par ailleurs, il nous a semblé intéressant de rapporter les comptages effectués sur le matériel éventuellement contaminé à la radioactivité moyenne par abeille et d'exprimer ainsi par un pourcentage que nous appelons indice de contamination, la valeur des erreurs imputables à ce facteur au cours de recherches utilisant la méthode des radio-isotopes.

En effet, au cours de nos études nous sommes amenés à étudier la prise de nourriture d'abeilles au niveau de l'individu, il est donc intéressant de savoir ce que représente la contamination du milieu ambiant par rapport à la radioactivité d'un individu. Mais les pourcentages ainsi obtenus doivent être divisés par un facteur de l'ordre de vingt pour exprimer la contamination réelle, c'est-à-dire, la quantité de radio-isotopes qui ne se trouve pas à l'intérieur du corns des abeilles.

\section{RÉSULTATS}

\section{I. - Contamination du matériel par des abeilles marquées avec de la nourriture liquide}

Nous savons que les abeilles sont en perpétuel mouvement à l'intérieur de la colonie et que les occasions de contacts corporels sont nombreuses.

Nous savons également que les abeilles vivent étroitement accolées les unes aux autres et parfois même amassées surtout lorsqu'elles forment, à certaines époques de l'année, ce qu'on appelle "la grappe ".

Nombreuses, bien sûr, sont les occasions de contacts externes; aussi nous sommes-nous posé cette question : " $\mathrm{Y}$ a-t-il un risque de contamination externe chez l'abeille ou dans un lot d'abeilles marquées au moyen de radioéléments, et par le fait même, si l'abeille est contaminée extérieurement souille-t-elle le milieu dans lequel elle vit? "Nous avons essayé de répondre à cette question, ou tout au moins d'en éclaircir certains points.

Nous avons, dans une première expérience, nourri un lot de Ig abeilles avec du sirop marqué au phosphore-32. Ces abeilles étaient réunies dans une cagette et la nourriture sucrée distribuée ad libitum. Dans ces conditions de nourrissement (durée 3 jours), nous étions sîrs que les abeilles ne consommaient que de la nourriture marquée; les risques de contamination étaient donc fortement amplifiés.

Au bout de 3 jours, les abeilles étaient soumises individuellement à un examen sous le compteur, ainsi que les différents éléments de la cagette,

Nous avons obtenu pour I9 abeilles :

- 50 794 coups; moyenne par abeille : 2673 coups par minute. 
I)ans le tableau I nous avons consigné les résultats de ce premier essai.

Nous avons signalé que le marquage avait duré 3 jours consécutifs. Au bout de ces 3 jours, une fois le comptage terminé, les abeilles étaient changées de cagette et nourries cette fois avec du candi non marqué (la nourriture solide étant plus pratique et mieux tolérée par des abeilles claustrées). En 24 h, nous n'avons enregistré, pour le candi et pour le lot d'abeilles, que i 3 c.p.m. ou 4 p. roo de contamination par rapport à la radioactivité moyenne d'une seule abeille.

TABLEAU I

\begin{tabular}{|c|c|c|}
\hline Nature du matériel & $\begin{array}{l}\text { Nombre de ch/s } \\
\text { au compteur GM }\end{array}$ & $\begin{array}{c}\text { Indice de } \\
\text { contamination }(\%)\end{array}$ \\
\hline $\begin{array}{l}2 \text { vitres } \ldots \ldots \ldots \ldots \\
\text { Languette de cire } \ldots \ldots \ldots\end{array}$ & $\begin{array}{r}91 \\
227\end{array}$ & $\begin{array}{l}3 \\
8\end{array}$ \\
\hline & $\mathrm{ch} / \mathrm{s}$ intégrateur & \\
\hline Cagette et abreuvoir .... & moins de $/ \mathrm{ch} / \mathrm{s}$ & \\
\hline
\end{tabular}

Cette contamination est due sans doute à des rejets de liquide provenant du jabot ou encore de la pollution de la langue.

Le candi était de nouveau changé et, $48 \mathrm{~h}$ après, soumis à un comptage. Nous n'avons alors enregistré que i 7 coups pour l'ensemble du lot.

Nous avons attendu I9 jours à partir du ${ }^{\text {er }}$ comptage avant d'en refaire un second. Les résultats sont consignés dans le tableau I bis. Deux abeilles étant mortes en cours d'expérience, nous avons obtenu pour I 7 abeilles I 6922 coups, soit 995 coups pour une abeille.

TABLEAU I bis

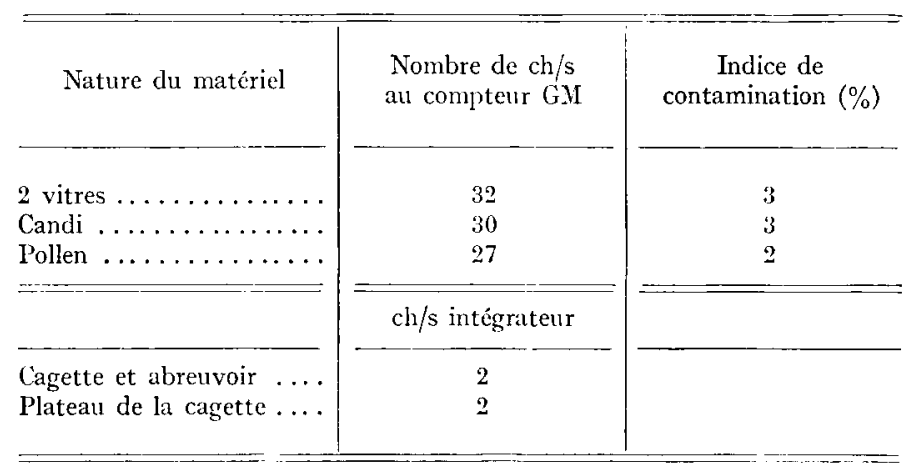

Ians cette prenière expérience, nous voyons donc que la contamination externe de l'abeille marquée avec du liquide est infime. Le matériel sur lequel et dans lequel elle vit n'est que très partiellement contaminé et ceci même après Ig jours de vie claustrée. 


\section{II - Contamination du matériel par les abeilles nourries aíec de la nourriture solide}

Dans une cagette, nous avons claustré 20 abeilles nourries avec du candi à teneur élevée en radiophosphore pour augmenter les chances de contamination.

Je souligne que, dans ce type de nourrissement les abeilles avaient plus facilement accès à la nourriture que dans l'autre cas. Le candi était déposé dans une boîte de matière plastique de $4 \times 2,7 \mathrm{~cm} \times 7 \mathrm{~mm}$ de haut.

La prise de nourriture a duré 3 jours consécutifs puis les abeilles ont été comptées ainsi que le matériel. L,e tableau 2, consigne les résultats de cet essai. I,e nombre de coups pour 20 abeilles était de 183905 , soit 9 I95 coups pour une abeille.

\section{TABLEAU 2}

\begin{tabular}{|c|c|c|}
\hline Nature du matériel & $\begin{array}{l}\text { Nombre de } \mathrm{ch} / \mathrm{s} \\
\text { au compteur GII }\end{array}$ & $\begin{array}{c}\text { Indice de } \\
\text { contamination }(\%)\end{array}$ \\
\hline $\begin{array}{l}2 \text { vitres } \ldots \ldots \ldots \ldots \ldots \ldots \\
\text { Cire } \ldots \ldots \ldots \ldots \ldots \ldots\end{array}$ & $\begin{array}{l}430 \\
8 \mathbf{1} 0\end{array}$ & $\frac{2}{4}$ \\
\hline & Ch/s intégrateur & \\
\hline Cagette et abreuvoir & 2 & pour la totalité du lot \\
\hline
\end{tabular}

Comme précédemment, après le comptage, les abeilles étaient changées de cagette et recevaient du candi " normal ». Deux jours après, un comptage du candi normal a été effectué, pour voir s'il y a eu contamination par rejets. Nous avons enregistré Igo coups soit environ o, I p. Ioo de la radioactivité totale et $2 \mathrm{p}$. Ioo de la radioactivité moyenne d'une abeille. On peut remarquer que la pollution, ici, n'est pas plus importante que dans l'autre essai.

Nous avons attendu I 6 jours à partir du premier comptage avant de procéder

TABI,EAU 2 bis

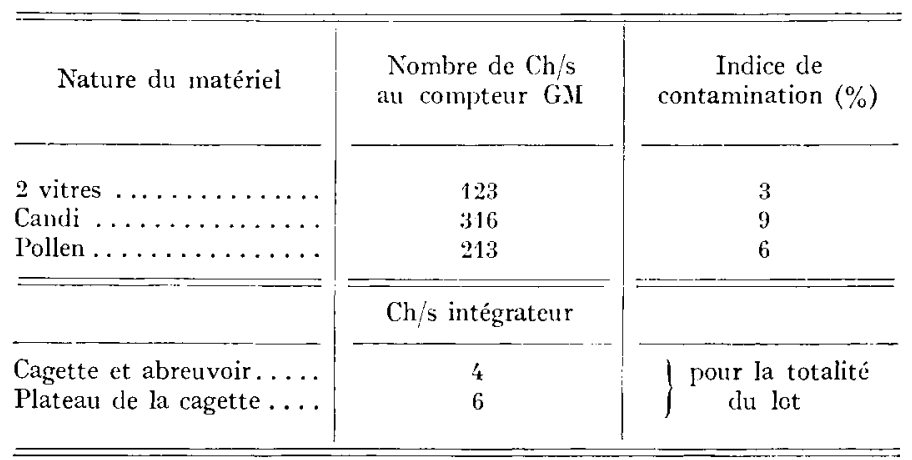


au second comptage dont le tableau 2 bis donne les résultats. Nous avons obtenu pour I9 abeilles survivantes, une étant morte en cours d'expérience, $6255^{8}$ coups, soit 3292 coups en moyenne pour une abeille.

Dans ce dernier cas, bien que la contamination du matériel ait été très légèrement plus importante que dans l'essai $n^{\circ} \mathrm{I}$, nous la considérons comme négligeable ; d'autant plus que dans l'essai $n^{\circ} 2$, les abeilles étaient au départ très fortement radioactives. 'Toutefois, nous avons enregistré 6 chocs seconde pour le plateau de la cagette contre 2 chocs seconde dans l'essai $n^{0}$ I. Cette radioactivité correspond sans doute à des particules de candi qui jonchaient la cagette et que les abeilles avaient transportées avec leurs poils lors du transfert de milieu après le premier comptage. Si l'on nettoie soigneusement le plateau de la cagette afin de le débarrasser des particules de candi marqué, la radioactivité disparaît totalement ; il n'y a pas d'absorption de la part du plateau de bois.

\section{III. - Contamination radioactive des téguments de l'abeille}

Iitant donné les résultats acquis par les premières expériences sur la contamination du matériel, il ne semble donc pas que l'abeille soit fortement contaminée " extérieurement " et qu'elle puisse souiller son milieu.

Pour préciser cette contamination nous nous sommes, cette fois, intéressé à l'abeille elle-mêne et nous avons étudié certaines parties des téguments.

I)ans cette expérience, nous avons mesuré la radioactivité des ailes, des 6 pattes, de la tête, du thorax et de l'abdomen.

Nous avons réuni dans une cagette 20 abeilles d'âge indéterminé. Ces abeilles ont été nourries avec du candi radioactif pendant 3 jours consécutifs. Ensuite, le candi marqué était retiré et les abeilles recevaient du candi normal.

Nous avons procédé, comme de coutume, à un premier comptage pour nous rendre compte de l'importance du marquage. Le lot des 20 abeilles réunissait 228807 coups, soit en moyenne I I 440 coups pour une abeille.

Tout au long de l'expérience qui a duré 9 jours, tous les deux jours, nous prélèvions quelques abeilles prises au hasard dans la cagette.

La radioactivité de l'abeille totale était comptée, puis celle-ci était sacrifiée. Ensuite, nous comptions à part successivement : les ailes, les pattes, la tête, le thorax et 1'abdomen. Ises différentes pièces étaient posées sur un papier changé entre chaque comptage pour éviter tout risque de contamination.

Le comptage des premières abeilles a commencé 24 h après le comptage de la totalité du lot, c'est-à-dire le lendemain du dernier jour de marquage.

Nous avons fait également un essai de lavage de l'abeille entière pour voir si l'abeille perdait un peu de sa radioactivité externe par immersion. Rappelons que SALI ERON (rg63), pour éviter une contamination générale au cours de dissections, a pratiqué celles-ci dans une solution de phosphate de sodium et n'a pas trouvé de radioactivité dans la solution.

Gossward et Krof'T ( $\mathrm{Ig}_{4}$ ) ont utilisé cette méthode pour éliminer une contamination externe éventuelle au cours d'études des échanges de nourriture chez les Fourmis du genre Formica. Les insectes étaient d'abord baignés dans une solution de phosphate de sodium, puis dans de l'eau pure.

Par la suite, nous avons remarqué qu'il n'était pas recommandé d'immerger 
totalement une abeille sous anesthésie. En effet, l'insecte endormi au $\mathrm{CO}_{2}$ dégurgite rapidement le contenu de son jabot et une goutte de sirop perle à l'extrémité de la langue.

La goutte de liquide peut alors, se dissoudre dans la solution constituant le bain et compromettre la manipulation.

Nous avons consigné les résultats de cette expérience dans le tableau 3. L,es chiffres correspondent aux moyennes des valeurs nominales enregistrées pendant toute la durée de l'essai.

TABLEAU 3

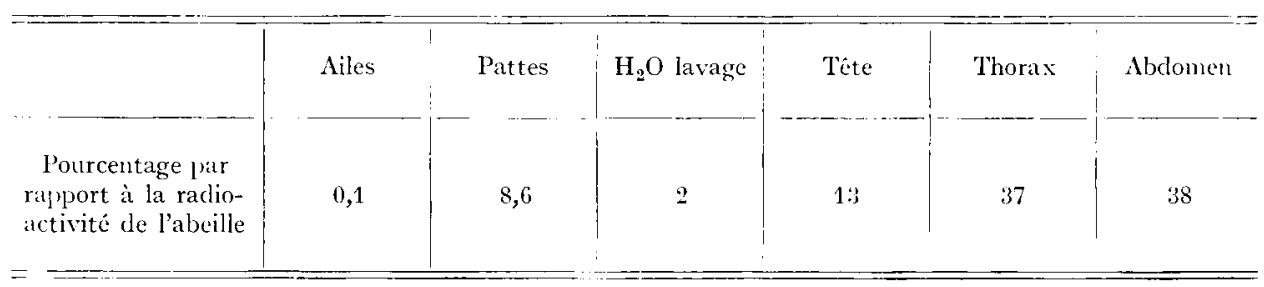

Nous pouvons remarquer la différence de la radioactivité des pièces énumérées et, par exemple, la pollution infime des ailes : $0, x$ p. Ioo.

Par contre, nous enregistrons 8,6 p. Ioo pour les pattes, I3 p. Ioo pour la tête et deux valeurs voisines, 37 et $38 \mathrm{p}$. Ioo, pour le thorax et l'abdomen; ces chiffres correspondant à la radioactivité du tube digestif, pour la plus grande part.

La solution de lavage n'a pas entraîné ou dissout de poussières provenant du thorax, pourtant fortement poilu, ou de 1'abdomen et sa radioactivité est seulement égale à 2 p. Ioo de celle de l'abeille.

A première vue, la radioactivité des pattes $(8,6 \mathrm{p}$. Ioo) pouvait être đue à une certaine activité externe, les pattes des abeilles étant directement en contact avec la nourriture.

Un petit dispositif à l'intérieur de la cagette a permis de faire une remarque importante, à notre avis, à ce sujet.

Nous avons disposé, dans une cagette, un double fond constitué par un grillage métallique à fines mailles. Les abeilles étaient obligées de marcher sur le grillage pour se déplacer et ainsi leurs pattes s'accrochaient dans les mailles et se nettoyaient. Nous avons appelé ce dispositif "le paillasson ". Sous le paillasson était placé un papier qui récupérait les poussières et les particules de candi qui se détachaient des pattes des abeilles.

Dans cet essai, les abeilles étaient nourries également au candi marqué et pendant 3 jours.

A la fin du nourrissement, les pattes des abeilles furent soumises au comptage et nous avons pu constater que l'indice moyen de contamination était de 7 p. roo. Le lot comprenait ro abeilles.

I,e "paillasson " était propre et nous avons compté les "poussières " tombées dans le papier. Il faut considérer deux sortes de " poussières " :

I $^{\circ}$ celles provenant de l'abeille elle-même ;

$2^{\circ}$ celles acquises par l'abeille et provenant de la nourriture qui s'est fixée aux pattes. 
Nous devons signaler que le sucre candi ne se fixe guère longtemps aux pattes, très vite la particule sucrée sèche, durcit et tombe. On peut voir régulièrement dans les cagettes d'abeilles nourries au candi, une grande quantité de fragments de sucre sur le plateau, que l'on prélève ordinairement lors du nettoyage de la cagette.

Après séparation sur le papier des particules sucrées, les poussières d'abeilles n'étaient pas radioactives, juste avant le comptage des pattes, nous avons brossé celles-ci avec un pinceau pour recueillir les poussières les plus agglutinées.

Cette expérience nous a éclairé sérieusement et imméđiatement nous avons étuđié plus complètement la contamination des pattes chez des insectes nourris : $\mathrm{I}^{\mathrm{o}}$ au solide ; $2^{\circ}$ au liquide.

\section{Contamination des pattes avec la nourriture solide.}

Nous avons attribué les 7 p. Ioo de radioactivité des pattes à une contamination interne. Les abeilles avaient consommé le candi pendant 3 jours, ce qui peut permettre au radio-isotope de se fixer à l'intérieur des différents organes.

Cette fois, un lot de $\mathrm{I}_{4}$ abeilles n'a reçu le candi marqué que pendant $\mathrm{I}_{2} \mathrm{~h}$. et a été immédiatement " compté ».

Dans ce cas, le pourcentage de radioactivité des pattes par rapport à la radioactivité totale de l'abeille était de 2 p. Ioo.

\section{Contamination des pattes avec la nourriture liquide.}

L'essai a été traité de la même façon que le précédent. I,e lot comprenait également I4 abeilles et la nourriture liquide était distribuée suivant la technique habituelle.

Jans ces conditions, nous n'avons enregistré en examinant les pattes que I,I p. Ioo de la radioactivité totale des insectes.

Dans ces deux essais, nous remarquons le faible pourcentage de contamination des pattes. Cette radioactivité est due sans doute à des fragments de sucre marqué, collés aux pattes de 1'abeille, celles-ci n'ayant pas été nettoyées avant le comptage dans le cas du nourrissement au candi. Dans le cas du marquage au sirop, la souillure des pattes en contact direct du liquide est fort peu probable d'après ce que nous avons pu remarquer, mais il faut considérer que le léchage des pattes chez l'insecte étudié peut être une source de pollution.

\section{CONCLUSION}

Nous pensons que le présent travail apporte quelques indications intéressantes sur le problème de la contamination externe de l'abeille et de son milieu au cours de recherches effectuées au moyen de radio-isotopes. Nous remarquons par les résultats acquis que, dans les deux cas de nourrissement, le pourcentage de pollution reste très faible quant aux organes les plus exposés extérieurement. Le biotope imposé aux abeilles pour plusieurs jours (cagettes de claustration) n'a pas en conséquence à souffrir d'une contamination trop importante.

Toutefois, il est utile de prêter attention au mode de distribution de la nourriture. 
Dans le cas du marquage au liquide sucré, seule la langue des abeilles doit avoir accès au sirop, un récipient trop large risquerait fort de compromettre la manipulation.

Le candi doit avoir une consistance convenable, c'est-à-dire un peu dur, mais jamais poisseux.

I'autre part, tenant compte de ces renseignements, la décontamination totale du matériel après expérience devrait être plus aisée.

Reşu pour publication en nö̀embre 1965.

\title{
SUMMARY
}

\begin{abstract}
STUDY OF THE EXTERNAL CONTAMINATION OF THE BEE AND ITS ENVIRONMENT
\end{abstract} BY MEANS OF A RADIOISOTOPE INTRODUCED INTO THE NLTRIMENT

The author studied the external pollution of the bee when fed either on solid nutriment (royal candy) or liquid nutriment (sugar syrup) marked with the isotope $\mathrm{I}^{32}$ in the form of sodium phosphate.

The results obtained show that, contrary to expectation, the external contamination of the bee was slight in both cases. The contamination of the small cages in which the bees lived during the whole of the experiment was equally slight. Examination of the different organs, in particular the legs, showed clearly that the radioactivity was localised in the interior of the body of the insect.

\section{RÉFÉRENCES BIBLIOGRAPHIQUES}

Courtols G., Lecomte J., 1958. Sur un procédé de marquage des abeilles butineuses au moyen d'un radio-isotope. C.R. Acall. Sci., 24\%, I47-I49.

Delvert-Salleron F., 1963. Étude au moyen de radio-isotopes, des échanges de nourriture entre reines, mâles et ouvrières d'Apis mellifica L. Ann. Abeille, 6, 3 , 20I-227.

Nixox H. L., Ribbasos C. R., I952. Food transmission within the honeybee community. Proc. roy. Soc. 140, $43-50$ B.,

Oertel J., Emerson R. B., Wheeler II. E., 1953. Transfer of radioactivity from worker to drone honcy bees after ingestion of radioactive sucrose. Ann. entomol. Soc. Amer., 46 (4). 